\title{
Radiofrequency neurotomy for the treatment of third occipital headache
}

\author{
J Govind, W King, B Bailey, N Bogduk
}

J Neurol Neurosurg Psychiatry 2003;74:88-93

See end of article for authors' affiliations .....................

Correspondence to: Professor N Bogduk, Department of Clinical Research, Royal Newcastle Hospital, Newcastle, NSW 2300, Australia; mgillam@ mail.newcastle.edu.au

Received 26 March 2002 In revised form 3 September 2002 Accepted 19 September 2002

\begin{abstract}
Objective: To evaluate the efficacy of a revised technique of percutaneous radiofrequency neurotomy for third occipital headache.

Methods: The revisions included using a large gauge electrode, ensuring minimum separation between the three electrode placements, and holding the electrode in place by hand. The revised technique was used to treat 51 nerves in 49 patients diagnosed as suffering from third occipital headache on the basis of controlled diagnostic blocks of the third occipital nerve. The criteria for successful outcome were complete relief of pain for at least 90 days associated with restoration of normal activities of daily living, and no use of drug treatment for the headache.

Results: Of the 49 patients, $43(88 \%)$ achieved a successful outcome. The median duration of relief in these patients was 297 days, with eight patients continuing to have ongoing relief. Fourteen patients underwent a repeat neurotomy to reinstate relief, with $12(86 \%)$ achieving a successful outcome. The median duration of relief in these patients was 217 days, with six patients having ongoing relief. Side effects of the procedure were consistent with coagulation of the third occipital nerve and consisted of slight ataxia, numbness, and temporary dysaesthesia. No side effects required intervention, and they were tolerated by the patients in exchange for the relief of headache.

Conclusions: Use of the revised procedure greatly improved the rather low success rate previously encountered with third occipital neurotomy. Although the relief of headache is limited in duration, it is profound and can be reinstated by repeat neurotomy. No other form of treatment has been validated for this common form of headache.
\end{abstract}

$\mathrm{E}$ xperimental studies on normal volunteers have indicated that referred pain from the C2-3 zygapophysial joint is perceived in the head. ${ }^{1}$ Clinical studies ${ }^{23}$ have shown that this form of headache can be relieved by anaesthetising the third occipital nerve, which is the nerve that innervates the C2-3 zygapophysial joint. ${ }^{4}$ For this reason, this form of headache has been named "third occipital headache." ${ }^{356}$ Among patients with whiplash injuries, third occipital headache is common, with a prevalence of $27 \% .^{3}$ In those patients in whom headache is the dominant complaint, its prevalence is $53 \%$ (with $95 \%$ confidence intervals of $37 \%$ to $68 \%$ ). ${ }^{3}$

Although common, third occipital headache has, to date, lacked a valid treatment. No studies have shown that it can be successfully treated with drugs or physical therapy. ${ }^{78}$ One uncontrolled study has reported that some patients can benefit, at least temporarily, from intra-articular injections of corticosteroids. ${ }^{9}$

Previous studies have shown that cervical zygapophysial joint pain, at levels below C2-3, can be successfully treated with percutaneous radiofrequency medial branch neurotomy. A randomised, double blind, controlled trial showed that the results of radiofrequency neurotomy were not a placebo effect. ${ }^{10}$ That study and subsequent observational studies ${ }^{11-13}$ have shown that patients treated by radiofrequency neurotomy can achieve complete relief of pain that is enduring; and although pain returns when the coagulated nerve recovers, it can be reinstated by repeating the neurotomy. ${ }^{12}$

Only one study has addressed the treatment by radiofrequency neurotomy of pain stemming from the C2-3 zygapophysial joint. ${ }^{14}$ It reported only modest response rates, and warned that radiofrequency neurotomy should not be used for the treatment of headache unless and until the technical problems associated with this procedure at $\mathrm{C} 2-3$ were overcome.

In the present study we report a revised method of radiofrequency neurotomy of the third occipital nerve and the results that can be obtained for the treatment of third occipital headache. These results serve to lift the embargo previously placed on the use of radiofrequency neurotomy for this condition.

\section{METHODS}

The study was conducted in a pain clinic specialising in the management of spinal pain, and located in a teaching hospital in the city of Newcastle, Australia. The clinic operates for two days a week and draws patients by referral from specialists and general practitioners in Newcastle, the city of Sydney (located some $170 \mathrm{~km}$ to the south), and throughout the state of New South Wales. Some patients were seen from other states in Australia, one from New Zealand, and one from Ireland.

During the period July 1998 to September 2001 the clinic saw some 120 patients whose cardinal complaint was headache associated with neck pain or a history of neck injury. These patients constituted the source population. All underwent third occipital nerve blocks, using standard techniques, ${ }^{315} 16$ under double blind, controlled conditions. These involved injecting $0.3 \mathrm{ml}$ of a local anaesthetic agent, under fluoroscopic control, onto each of three target points for the third occipital nerve. Controlled blocks were used in order to rule out false positive responses which commonly occur when only single diagnostic blocks are used..$^{15}{ }^{16}$ The response to a block was assessed independently by a nurse who was not involved in the performance of the block.

For their first block, patients were randomly assigned to receive either lignocaine $2 \%$ or bupivacaine $0.5 \%$. Patients whose headache was not relieved by this block were considered not to have third occipital headache, and went on to other investigations or management. They were not included in the present study. Those patients who responded to the first block returned for a control block, using the local 
anaesthetic agent that was not used for their first block. Patients were considered to have a positive response if on each occasion that the nerve was blocked they obtained complete relief of their headache, and provided that the duration of relief obtained when bupivacaine was used (typically three to five hours) was longer than that obtained when lignocaine was used (typically one to two hours)..315 16

In all, 49 patients satisfied these diagnostic criteria for third occipital headache. They became the study sample. All were offered third occipital neurotomy, and all consented. In 47 patients the symptoms were unilateral and treatment was undertaken on the side of the pain. Two patients had bilateral pain, and satisfied the diagnostic criteria for third occipital headache on both sides. Each side was treated strategically on separate occasions.

Third occipital neurotomy was performed under sterile conditions, with the patient lying in a lateral position with the target side uppermost. The procedure was done under fluoroscopic control and under local anaesthesia. This involved anaesthetising the third occipital nerve with $1-2 \mathrm{ml}$ of $0.5 \%$ bupivacaine, at the site where it crossed the lateral aspect of the C2-3 zygapophysial joint. In addition, the puncture sites and tracks for the electrode used were anaesthetised with a total of about $4 \mathrm{ml}$ of $2 \%$ lignocaine. No sedation or other anaesthesia was required or used. Once the third occipital nerve had been anaesthetised, the spinal needle used for the injection was left in place, resting on the target site, in order to facilitate the introduction of the electrode.

Neurotomy was undertakne using a Ray electrode (Radionics, Burlington, Massachusetts, USA). In order to coagulate the third occipital nerve thoroughly, multiple lesions were produced along each of two passes. For the first pass, the electrode was introduced along an oblique plane, $30^{\circ}$ from the sagittal plane, passing ventrally and medially. In this plane a puncture point in the skin was selected directly over the target site marked by the needle used for the nerve block. This puncture point was pierced using a No 11 scalpel blade, in order to allow penetration of the electrode through the skin. The electrode was passed slowly through the skin and posterior neck muscles towards the target point, using repeated posteroanterior fluoroscopic imaging to guide its passage. Initially it was aimed to strike the posterior aspect of the C2-3 zygapophysial joint. This measure guards against the electrode being inserted too deeply. Once the electrode had contacted the back of the C2-3 joint, it was carefully redirected to slip a few millimetres across the lateral surface of the joint. Subsequently, the further insertion of the electrode was monitored using lateral views. One of three target positions was sought: opposite the lower third, the middle third, or the upper third of the superior articular process of C3. Three target points were used in order to accommodate possible variations in the course of the third occipital nerve, which most commonly crosses the middle of the $\mathrm{C} 3$ articular pillar but can range as high as the tip of the superior articular process or as low as the base of that process. ${ }^{11}$

A further guideline was followed: as the electrode was systematically placed at each of the target points, it should be located no further than two electrode diameters from where the nearest adjacent electrode had been placed (fig 1). This criterion meant that, if all electrode placements were reconstructed on the same lateral view of the C3 articular pillar, the gap between adjacent electrodes was not greater than the width of one electrode. This measure ensured that adjacent lesions were not only contiguous but overlapped, with no region between electrode placements that could escape coagulation. This guideline was occasionally modified. In patients with tall articular pillars, four rather than three positions were used, in order to satisfy the criterion for contiguous lesions. In patients with short articular pillars, the criterion could be satisfied with two placements.

For each position, the electrode was placed so that its tip was palpably in contact medially with the surface of the pillar and
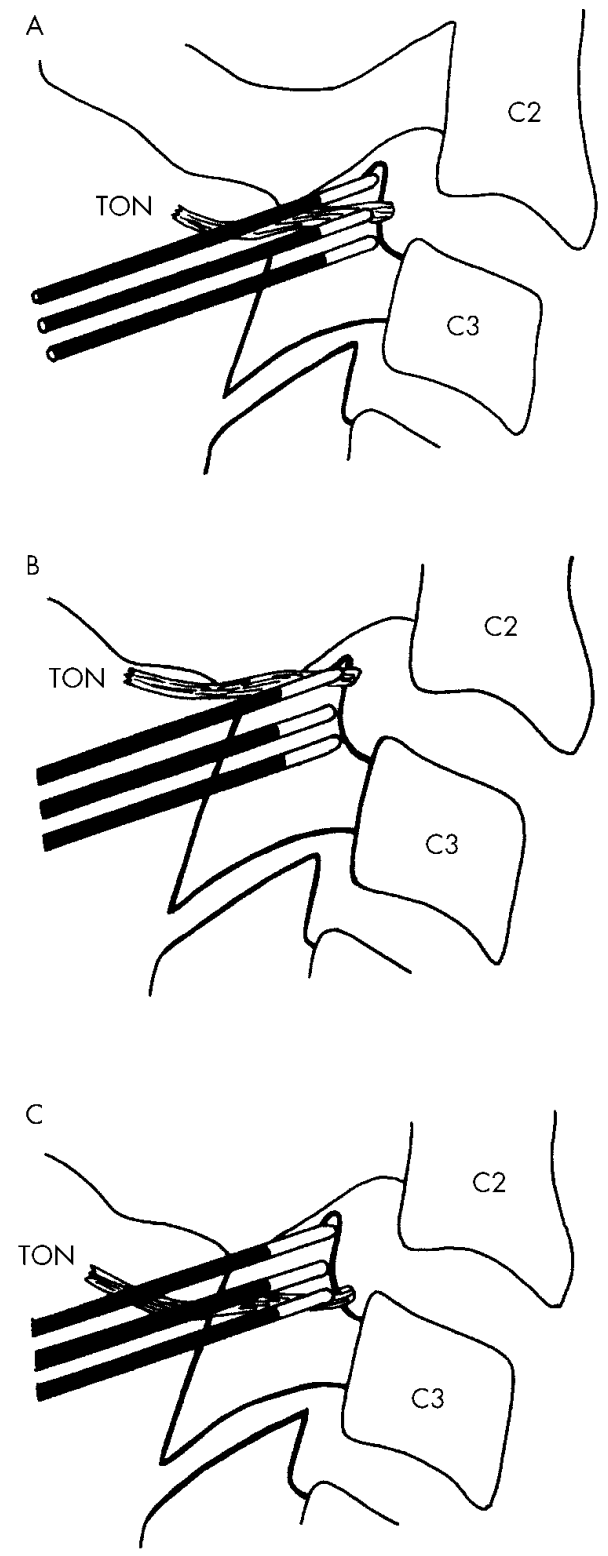

Figure 1 A sketch showing how the electrode should be placed in three locations across the superior articular process of $\mathrm{C} 3$, such that each placement lies no further than one electrode diameter from the adjacent placement, in order that the third occipital nerve does not escape coagulation. (A) The most common course of the nerve. (B) A high location of the nerve. (C) A low location of the nerve. C2, C2 vertebral body; C3, C3 vertebral body; TON, third occipital nerve.

reached no further than the anterior margin of the pillar, as seen in lateral fluoroscopic views (fig 2A). On posteroanterior views the rounded tip of the electrode would project just medial to the lateral border of the radiographic silhouette of the pillar (fig $2 \mathrm{~B})$. Oblique views were used to ensure that the tip of the electrode was in contact with bone medially. (In this position, the electrode rests on the anterolateral surface of the articular pillar and does not project into the intervertebral foramen; it is displaced several millimetres from the spinal nerve and ventral ramus, which therefore are not vulnerable to coagulation.)

Once the electrode had been placed in correct position and its position confirmed radiographically, it was carefully held in place, and a lesion was generated. The lesion was made by raising the temperature of the electrode, at a rate of $1^{\circ} \mathrm{C}$ per second, until it reached $80^{\circ} \mathrm{C}$. The temperature was maintained for 90 seconds, at a level not less than $80^{\circ} \mathrm{C}$ and not 

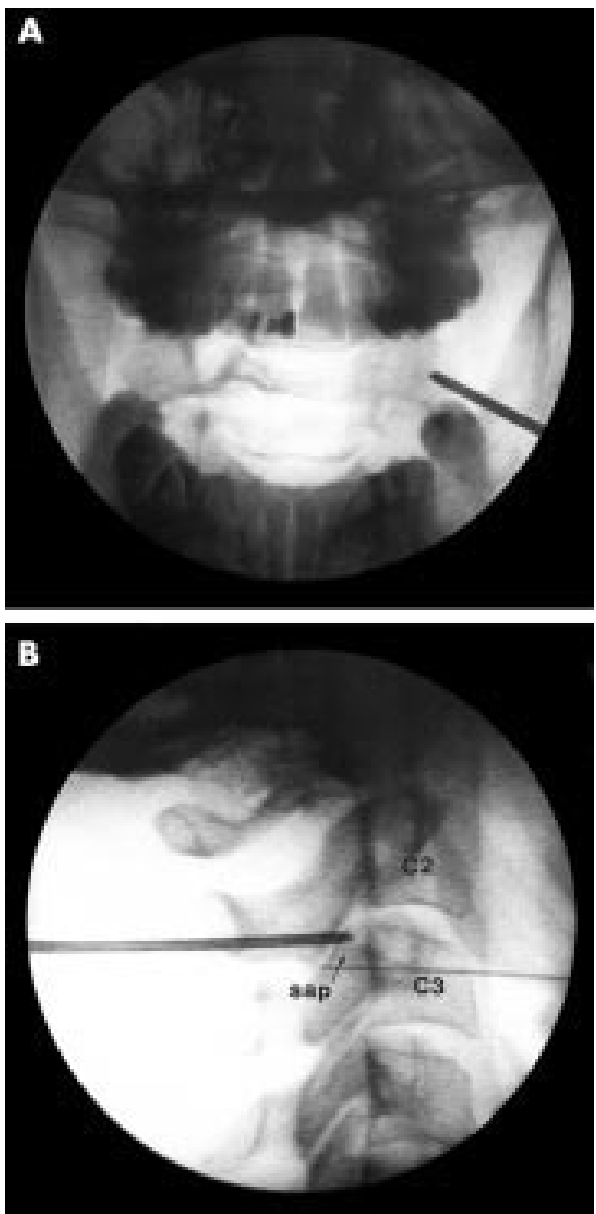

Figure 2 Radiographs showing the correct placement of an electrode along an oblique pass to coagulate the third occipital nerve. (A) Anteroposterior view. (B) Lateral view. C2, C2 vertebral body; C3, C3 vertebral body; sap, superior articular process of C3.

greater than $85^{\circ} \mathrm{C}$. Once the lesion had been made at the first target point selected, the electrode was readjusted to one of the other two target points. The same precautions and checks were taken to ensure correct placement of the electrode at the new position, and a lesion was made using the same parameters. Once the second lesion had been made, the electrode was readjusted to the third, remaining position, and the procedure repeated to make a third lesion. Where required, a fourth lesion was made, in accordance with the guidelines for electrode placement.

A second series of lesions was made along a sagittal plane. A puncture point was selected and pierced overlying the lateral aspect of the $\mathrm{C} 2-3$ joint, as seen in a posteroanterior view. As before, the electrode was introduced, first to strike the back of the C2-3 joint, and then adjusted to slip across its lateral aspect. Under lateral fluoroscopic views, the electrode was adjusted to one of three positions (or four or two, according to the size of the C3 articular pillar). Those positions were opposite the superior, middle, and inferior thirds of the C3 articular pillar. In each position, the electrode was placed so that medially it was in contact with bone, and its uninsulated portion was centred over the posteroanterior centre of the C2-3 joint, as seen on lateral views (fig 3A). Posteroanterior views were used to check that the electrode was in contact with bone (fig 3B). At each of the three positions in the sagittal pass, a lesion was made at $85^{\circ} \mathrm{C}$ for 90 seconds.

In both the oblique and sagittal passes, particular attention was paid to holding the electrode steady in each position, as each lesion was made. At the start of lesioning and at approximately 30 second intervals while the lesion was being made, the
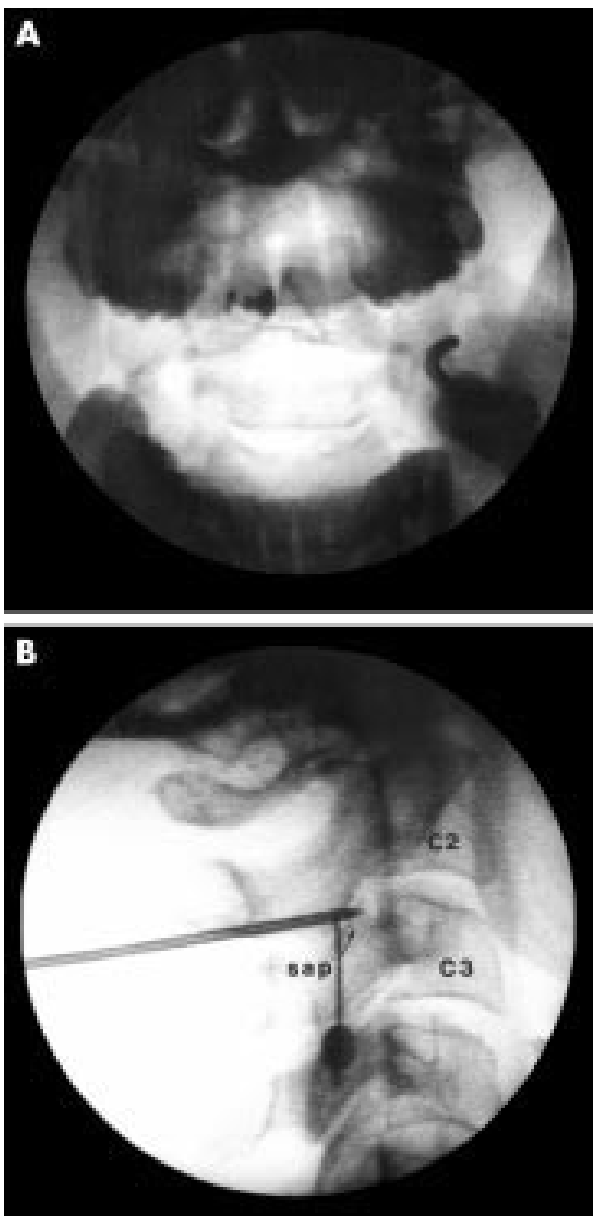

Figure 3 Radiographs showing the correct placement of an electrode along a sagittal pass to coagulate the third occipital nerve. (A) Anteroposterior view. (B) Lateral view. C2, C2 vertebral body; C3, C3 vertebral body; sap, superior articular process of C3.

position of the electrode was checked using fluoroscopic screening. If the electrode had not moved, lesioning was continued. If the electrode had moved, lesioning was stopped; electrode position was re-established and confirmed on posteroanterior and lateral screening; and the lesioning started again.

At the conclusion of the procedure, $2 \mathrm{ml}$ of $0.5 \%$ bupivicaine were injected into the region where lesions had been made in order to provide postoperative analgesia. The puncture sites were washed with alcohol and dressed with a small, circular adhesive patch. Patients were prescribed a compound analgesic or oxycodone for later postoperative pain.

Following the operation, patients were reviewed by telephone contact on a weekly basis for the first month, and monthly thereafter. Patients were seen in person, if they so desired, and if they reported any untoward side effects or possible complications, so that these could be assessed, documented, and managed if required.

The primary outcome measure was complete relief of pain, such that the patient did not require any drug treatment or other treatment for their headache. For this purpose, the patient had only to assert that their headache was completely relieved, and that they had no remaining pain. This absolute criterion obviated the need to use visual analogue scales for measuring pain intensity. A secondary outcome was that the patient had resumed normal daily activities unaffected by headache. Other outcomes that were monitored and recorded were any side effects that the patient attributed to the operation.

A successful outcome was defined as complete relief of headache, associated with restoration of normal activities of 
Table 1 Demographic and clinical features of 49 patients with headache treated with third occipital neurotomy

\begin{tabular}{ll}
\hline Characteristic & \\
\hline Male patients ( $\mathrm{n}$ ) & 21 \\
Median age (years) & 41 \\
Interquartile range (years) & 33 to 47 \\
Female patients ( $\mathrm{n}$ ) & 28 \\
Median age (years) & 45 \\
Interquartile range (years) & 38 to 51 \\
Median pain intensity (0-100) & 80 \\
Interquartile range & 60 to 90 \\
Median duration of symptoms (months) & 24 \\
Interquartile range (months) & 12 to 66 \\
Range (months) & 6 to 240 \\
Compensation claim (n) & 33 \\
No compensation claim (n) & 16 \\
\hline
\end{tabular}

daily living, for a period initially lasting 90 days. Patients who did not obtain complete relief of headache were classified as failure, as were patients who obtained complete relief but not lasting for 90 days. For those patients achieving a successful outcome, the duration of complete relief was monitored.

For patients who had previously obtained complete relief of pain, but whose headaches recurred after 90 days, repeat neurotomy was offered. Patients who wanted the relief reinstated consented to repeat treatment. The results of these repeat neurotomies were monitored separately from the initial responses.

\section{RESULTS}

The demographic and clinical features of the patients treated are provided in table 1 . All suffered occipital headache, radiating to various extents into the parietal, frontal, or orbital regions of the head.

Six patients failed to satisfy the criteria for a successful outcome. Although four of these patients reported complete relief of pain initially, the relief did not last for the minimum period of 90 days. One of the six patients obtained no relief, and one was lost to follow up in the immediate postoperative period. At the time of writing, one of the six patients was waiting for a repeat neurotomy; two were seeking other care, and three could not be contacted. In all these patients the treatment was considered to have failed.

Forty three patients satisfied the criterion for a successful outcome, including the two who had bilateral neurotomies. Each patient achieved complete relief of pain for at least 90 days, and restored their normal activities of daily living. The initial success rate amounted to $88 \%$ (45 of 51 nerves, in 49 patients).

At the time of writing, eight of these patients had continuing complete relief of their pain. Fourteen patients ceased to have complete relief and underwent repeat neurotomy. Eight were awaiting repeat neurotomy. Headaches recurred in three patients, but not to a severity that they considered warranted repeat neurotomy; their pain was controlled by simple analgesics. Having recorded their initial response to treatment, two patients were lost to further follow up. Four patients initially had relief, but other medical conditions prevented them from pursuing repeat neurotomy. A further four patients with initially successful outcomes did not want repeat neurotomy, and sought other care for their headaches when they recurred.

The responses of these patients are depicted graphically in fig 4 and statistically in table 2 . The figure shows the duration of complete relief achieved by each patient, which patients underwent repeat neurotomy, and the duration of relief following each repeat. Numerically, the median duration of

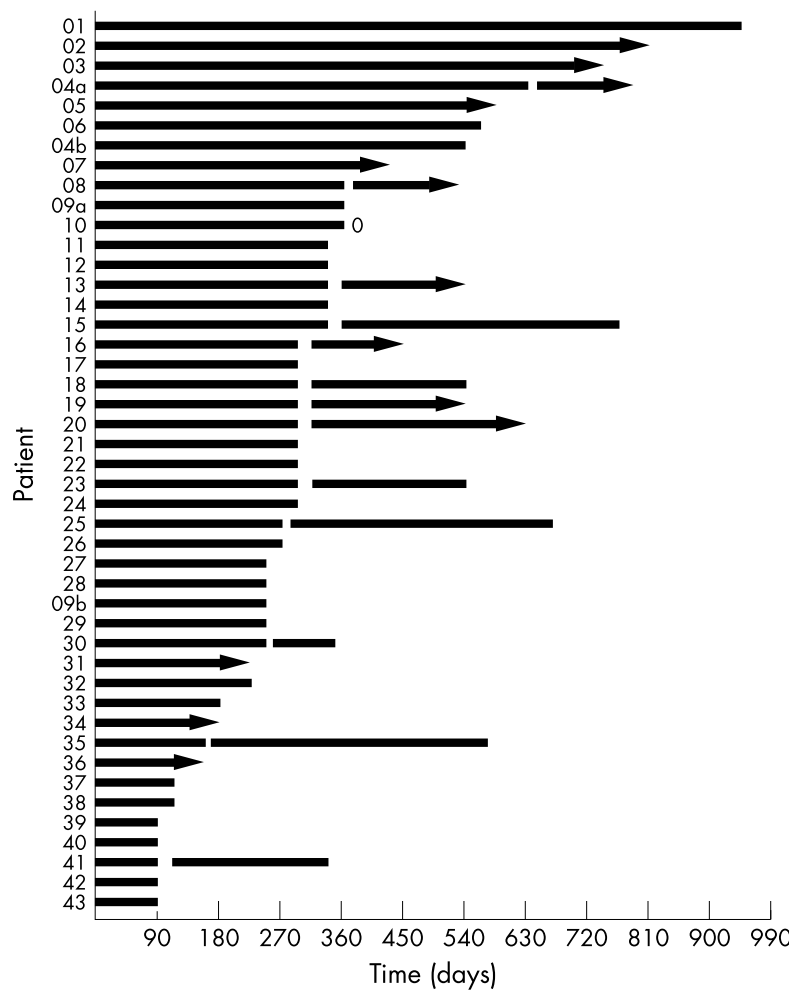

Figure 4 The duration of response of patients who obtained complete relief of headache following radiofrequency neurotomy. Arrowheads indicate ongoing relief. Interrupted bars indicate cessation of relief followed by the response to repeat neurotomy. " 0 " indicates no response to repeat neurotomy; " $a$ " and " $b$ " refer to the two patients who had bilateral neurotomies.

Table 2 Number of patients who achieved complete relief for the durations indicated, and the number in each category with continuing relief at the time of writing

\begin{tabular}{lll}
\hline $\begin{array}{l}\text { Duration of relief } \\
\text { (days) }\end{array}$ & $\begin{array}{l}\text { Achieving relief ( } \\
(\%))\end{array}$ & $\begin{array}{l}\text { Number with continuing } \\
\text { relief }\end{array}$ \\
\hline Failure & $6(13)$ & 0 \\
$<90$ & & \\
Success & $9(20)$ & 2 \\
91 to 150 & $2(4)$ & 1 \\
151 to 210 & $8(17)$ & 1 \\
211 to 270 & $11(24)$ & 0 \\
271 to 330 & $7(15)$ & 0 \\
331 to 390 & $1(2)$ & 1 \\
391 to 450 & $7(16)$ & 3 \\
$>510$ & 45 & 8 \\
Total &
\end{tabular}

The 45 successful neurotomies were undertaken in 43 patients.

complete relief of pain in those patients with a successful outcome was 297 days, with eight having ongoing relief.

Of the 14 patients who underwent repeat neurotomy, one obtained no relief, one achieved only 90 days of complete relief, but $12(86 \%)$ regained complete relief lasting beyond 90 days. The median duration of relief following repeat neurotomies was 217 days, with six patients still having ongoing relief at the time of writing (fig 4).

Litigation did not affect outcome. There were no statistically significant differences between patients with ongoing compensation claims and those who had no claim or who had already settled their claim, with respect to the proportion of 
Table 3 Lack of influence of litigation status on outcome of third occipital neurotomy

\begin{tabular}{|c|c|c|c|c|}
\hline Status & $\mathrm{n}$ & Success & Failure & $\begin{array}{l}\text { Duration of relief } \\
\text { (median days } \\
\text { (interquartile range)) }\end{array}$ \\
\hline Litigation & 33 & 28 & 5 & 249 (94 to 312) \\
\hline $\begin{array}{l}\text { No litigation } \\
\text { p Value }\end{array}$ & 16 & 15 & $\begin{array}{c}1 \\
373\end{array}$ & $\begin{array}{c}297(127 \text { to } 363) \\
0.299\end{array}$ \\
\hline
\end{tabular}

For success $v$ failure, the $p$ value relates to a $\chi^{2}$ test. For duration of relief, the $p$ value relates to a Mann-Whitney test.

\begin{tabular}{|c|c|}
\hline Side effect & Incidence \\
\hline Numbness & $97 \%$ \\
\hline Ataxia & $95 \%$ \\
\hline Dysaesthesia & $55 \%$ \\
\hline Hypersensitivity & $15 \%$ \\
\hline Itch & $10 \%$ \\
\hline
\end{tabular}

patients who had a successful outcome or the duration of relief that they obtained (table 3 ).

No complications were encountered, but there were several side effects (table 4). None of these side effects, however, was distressing enough to require intervention. When they occurred, dysaesthesia and hypersensitivity in the cutaneous territory of the third occipital nerve were self limiting and lasted only 7-10 days in most cases, and not longer than four weeks in one patient. None of the patients had sensory loss in the territory supplied by the ventral ramus of $\mathrm{C} 3$, or any other features that might indicate that this nerve had been affected by the surgery.

\section{DISCUSSION}

The results of the present study are substantially better than those reported by Lord et al, who achieved complete relief of pain in only $30 \%$ of their patients with third occipital headache. In the present study, the success rate was $88 \%$ following the first treatment, and $86 \%$ following repeat treatment. Several factors account for this.

First, the electrodes were carefully held in place while lesions were generated, and their position was monitored intermittently by fluoroscopy. If electrodes are not held in place, there is a risk of the electrode dislodging sufficiently to compromise the accuracy of the lesion placement. Movement of the patient, or muscle spasm, can expel the electrode dorsally; or, under the cantilever weight of the hub and cables attached to it, the electrode can sway away from the correct target site.

Second, Ray electrodes (Radionics) were used instead of SMK electrodes (also Radionics). The former are larger in diameter and make a lesion that is somewhat greater in size. This renders it less likely that the target nerve will escape coagulation when the electrode is not located exactly on the nerve.

Perhaps most significantly, attention was paid to ensuring that consecutive lesions were placed no more than one electrode width from an adjacent lesion. This measure ensures that no tissue located between electrode placements escapes coagulation. If electrodes are placed further apart than this, the possibility arises of a nerve escaping coagulation-or being incompletely coagulated-if it happens to run between two electrode placements. This same principle would apply if smaller electrodes are used, but in that event four or even five placements might be required in order to cover adequately the territory through which the third occipital nerve can run.

The present results are offered as those of an observational study. Placebo controls were not used for two reasons. First, as the third occipital nerve has a cutaneous distribution, patients could not be blinded to having a sham procedure. Second, but most important, radiofrequency neurotomy has already been subjected to a double blind, placebo controlled trial, which showed that its therapeutic results are not a placebo effect. ${ }^{10}$ As its effects are genuine at lower cervical levels, it is safe to surmise that they will be equally genuine on the third occipital nerve.

The results show that radiofrequency neurotomy can achieve complete relief of pain in a large proportion of patients diagnosed as having third occipital headache, on the basis of controlled diagnostic blocks. The relief is not permanent, for the nerve regenerates. This is to be expected, for unlike trigeminal radiofrequency neurotomy-in which the ganglion and cell bodies are targeted - third occipital neurotomy targets the peripheral axons of the nerve. As the cell bodies remain intact, the nerve should, and does, regenerate. Nevertheless, the periods of relief obtained are substantial and appear clinically significant. Moreover, relief can be reinstated by repeating the procedure. It is not known exactly how many repetitions patients can have without diminishing effect, for insufficient numbers of patients have been treated repeatedly. However, one patient has had seven repetitions that have succeeded in maintaining her pain-free for over eight years.

We have not ventured to perform neurotomy of the $\mathrm{C} 3$ dorsal root ganglion because we do not want to incur possible complications from denervation of the territory innervated by the $\mathrm{C} 3$ ventral ramus. Third occipital neurotomy restricts any complications to the territory of only the superficial medial branch of the $\mathrm{C} 3$ dorsal ramus. In that regard, the side effects encountered in the present study are ones that could and should be expected.

The third occipital nerve supplies a substantial proportion of the semispinalis capitis muscles. ${ }^{4}$ It is, thereby, involved in proprioception mediated by this muscle. Third occipital neurotomy inescapably partially denervates this muscle and so interferes with tonic neck reflexes. Ataxia is therefore to be expected. However, the patients in the present study were not disabled by the ataxia they suffered. It amounts to little more than an unsteadiness, particularly on looking downwards. The sensation is readily overcome by relying on visual cues: fixing on the horizon or horizontal features in the environment.

Numbness is also a predictable side effect because of the cutaneous distribution of the third occipital nerve. Indeed, the onset of numbness is a sign that the nerve has been successfully coagulated. Absence of numbness indicates a technical failure. Fortunately, the territory involved is small and not in a vital or critical area. Patients readily tolerate the numbness in exchange for the relief of pain they obtain.

Dysaesthesiae and hypersensitivity occur typically at the border of the area of skin innervated by the third occipital nerve. Ostensibly this reflects central disinhibition of adjacent cutaneous nerves that overlap into the third occipital territory. Although alarming at its onset, this dysaesthesia fortunately proved not to be distressing to the patients in the present study, and was short lived. Interestingly, loss of numbness and recurrence of dysaesthesia commonly preceded the recurrence of pain and could be used a cue that the third occipital nerve is recovering.

Third occipital neurotomy is neither a perfect nor an ideal solution for third occipital headache. It does not address or reverse the cause of pain-it is only a palliative procedure. A better treatment with longer lasting relief is required. However, at present there is no other form of treatment for this common form of headache. No drugs have been shown to relieve it, and intra-articular injection of corticosteroids affords only partial relief, for short periods, in only some 
patients. In contrast, third occipital neurotomy offers the prospect of complete relief of pain.

Some practitioners may be averse to implementing a treatment that requires repetition. Surgeons, in particular, may find it unsatisfying that a procedure does not effect a permanent cure. For these reasons, third occipital neurotomy is not promoted as a treatment that should be universally adopted. Indeed, the skills and patience required to perform it correctly would limit its appeal to aficionados with a special interest in this problem. However, the results of the present study indicate that a treatment is available for a condition that is common, has gone unrecognised, and for which no other treatment options have been, or are, available.

\section{Authors' affiliations}

J Govind, W King, B Bailey, N Bogduk, University of Newcastle, Department of Clinical Research, Royal Newcastle Hospital, Newcastle, Australia

Competing interests: none declared

\section{REFERENCES}

1 Dwyer A, Aprill C, Bogduk N. Cervical zygapophyseal joint pain patterns. I. A study in normal volunteers. Spine 1990;15:453-7.

2 Bogduk N, Marsland A. On the concept of third occipital headache. J Neurol Neurosurg Psychiatry 1986;49:775-80.

3 Lord S, Barnsley L, Wallis B, et al. Third occipital nerve headache: a prevalence study. J Neurol Neurosurg Psychiatry 1994;57:1 187-90.
4 Bogduk $\mathbf{N}$. The clinical anatomy of the cervical dorsal rami. Spine 1982;7:319-30.

5 Editorial. Third-nerve headache. Lancet 1986;ii:374.

6 Gobel H, Edmeads JG. Disorders of the skull and cervical spine. In: Olesen J, Tfelt-Hansen P, Welch KMA, eds. The headaches, 2nd ed. Philadelphia: Lippincott Williams \& Wilkins, 2000:891-8.

7 Bogduk N. Headache and the neck. In: Goadsby PJ, Silberstein SD, eds. Headache. Boston: Butterworth-Heinemann, 1997:369-81.

8 Bogduk N. Cervicogenic headache. In: Diener HC, ed. Drug treatment of migraine and other headaches. (Monographs in Clinical Neuroscience, vol 17.) Basel: Karger, 2000:357-62.

9 Slipman CW, Lipetz JS, Plastara CT, et al. Therapeutic zygapophyseal joint injections for headache emanating from the C2-3 joint. Am J Phys Med Rehabil 2001;80:182-8.

10 Lord SM, Barnsley L, Wallis BJ, et al. Percutaneous radio-frequency neurotomy for chronic cervical zygapophysial-joint pain. N Engl J Med 1996;335:1721-6.

11 Lord SM, McDonald GJ, Bogduk N. Percutaneous radiofrequency neurotomy of the cervical medial branches: a validated treatment for cervical zygapophysial joint pain. Neurosurg Q 1998;8:288-308.

12 McDonald G, Lord SM, Bogduk N. Long-term follow-up of cervical radiofrequency neurotomy for chronic neck pain. Neurosurgery 1999:45:61-8.

13 Sapir D, Gorup JM. Radiofrequency medial branch neurotomy in litigan and nonlitigant patients with cervical whiplash. Spine 2001; 26:E268-73.

14 Lord SM, Barnsley L, Bogduk N. Percutaneous radiofrequency neurotomy in the treatment of cervical zygapophysial joint pain: a caution. Neurosurgery 1995;36:732-9.

15 Bogduk N. International Spinal Injection Society guidelines for the performance of spinal injection procedures. Part 1: zygapophysial joint blocks. Clin J Pain 1997;13:285-302.

16 Bogduk N, Lord SM. Cervical zygapophysial joint pain. Neurosurg $Q$ 1998;8:107-17.

\section{NEURONLINE}

\section{The Neuromuscular Disease Center: www.neuro.wustl.edu/neuromuscular}

T

he website of the Neuromuscular Disease Center at Washington University School

of Medicine, St Louis, is quite simply an excellent online resource for all issues related to neuromuscular disorders. It is up to date, extensive and highly relevant to any busy clinical neurologist, academic, or basic scientist with an interest in neuromuscular disorders. It is written and edited by Alan Pestronk, who deserves much praise for his efforts. On several occasions, I have found this website of direct use in ways in which traditional meth ods are more limited (eg links to useful images for lectures or teaching, and assistance with differential diagnosis of rare congenital myopathies to name a few).

Some may dislike the terse bullet poin style or the lengthy, unmemorable URL (www.neuro.wustl.edu/neuromuscular). However, I suspect that they, like me, will be won over by the huge amount of useful content this site has to offer (differential diagnosis, links to laboratories for testing, and web links to everything from PubMed to patient information). In recent months, the site has improved further with an increasing number of images (eg photos of Duchenne de Boulougne, muscle hypertrophy, and muscle biopsy appearances in the section for Duchenne/Becker muscular dystrophy), which lighten the text somewhat. In summary, this is a site that I have much to commend and little to criticise, I hope you find it as useful as I have.

D J Nicholl

Department of Clinical Neurology, Queen Elizabeth Hospital, Edgbaston, Birmingham B15 2TH, UK

d.j.nicholl@bham.ac.uk

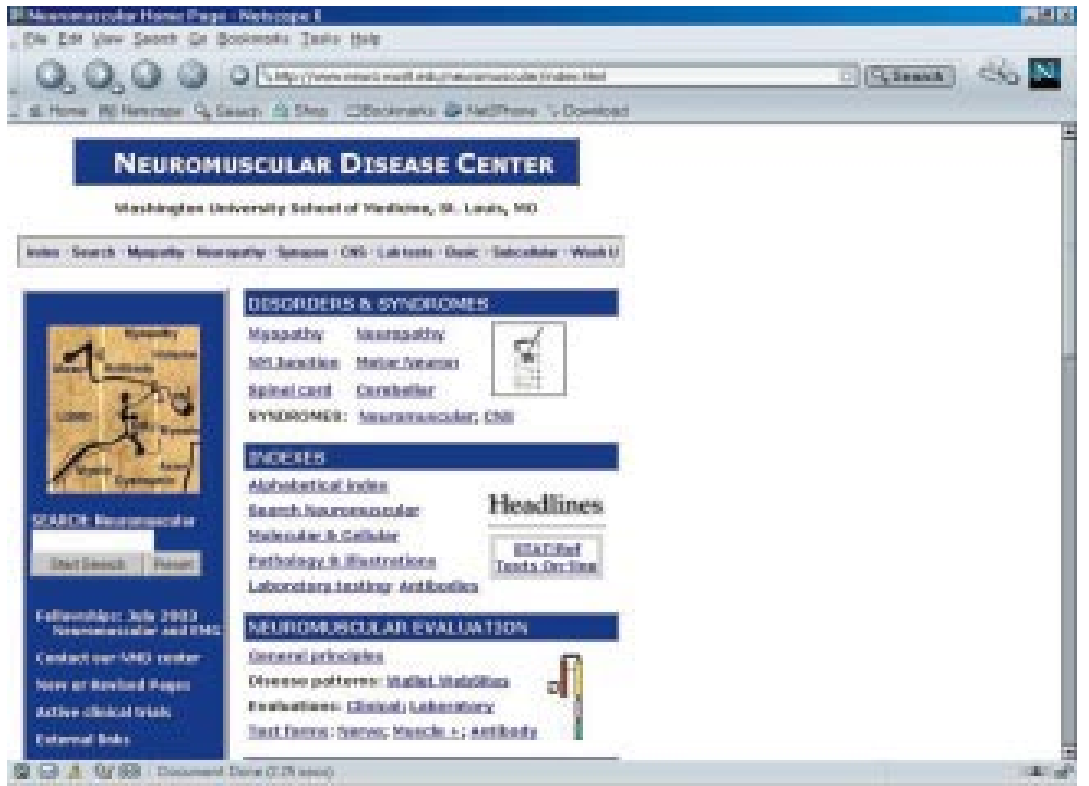

